\title{
Lapurdum
}

LAPURDUM Euskal ikerketen aldizkaria | Revue d'études basques |

Revista de estudios vascos | Basque studies review

$8 \mid 2003$

Numéro VIII

\section{Les Musulmans, le Pays Basque et la Navarre}

Yvette Cardaillac-Hermosilla

URL : http://journals.openedition.org/lapurdum/927

DOI : 10.4000/lapurdum.927

ISSN : 1965-0655

Éditeur

IKER

Édition imprimée

Date de publication : 1 novembre 2003

Pagination : 85-96

ISBN : 9782867813436

ISSN : $1273-3830$

\section{Référence électronique}

Yvette Cardaillac-Hermosilla, "Les Musulmans, le Pays Basque et la Navarre », Lapurdum [En ligne], 8 | 2003, mis en ligne le 01 juin 2009, consulté le 01 mai 2019. URL : http://journals.openedition.org/ lapurdum/927 ; DOI : 10.4000/lapurdum.927 


\section{Yvette Cardaillac - Hermosilla}

Université de Bordeaux III

\section{Les Musulmans, le Pays Basque et la Navarre}

Après la publication de divers articles sur la présence musulmane au Pays Basque et en Navarre, un panorama historique servant de cadre à ces travaux s'imposait. Nous nous sommes efforcés d'associer les informations émanant des deux civilisations, même si les sources sont de conception différente, pour mieux comprendre des manifestations qui perdurent des siècles après les expulsions.

Cependant nous sommes bien conscients que l'histoire des Musulmans de Navarre ne peut être dissociée de celles des Juifs, en effet ces deux minorités se concentrent dans le Sud et dans la vallée de l'Ebre. Les cartes de leur présence sur le terrain se superposent bien avec une plus grande diffusion de la population juive. Tous sont tributaires de leur environnement et des évènements généraux qui ont de grandes répercutions sur ces communautés.

Il existe un modèle de soumission dans l'Islam ${ }^{1}$ qui va se transmettre aux relations chrétiennes Musulmanes après la reconquête. En échange d'une promesse de fidélité, de tribus et d'otages, les habitants se trouvaient sous la protection de l'Islam. La dépendance imposait l'acceptation d'une superstructure politique, mais permettait aux habitants de conserver leurs propriétés, traditions juridiques, religieuses et culturelles sous l'autorité locale. Le cas se produit dans les plaines d'Alava où l'on réclame en 767 les tributs non payés. ${ }^{2}$

La sanction économique réelle imposée aux dhimmis (soumis) est de nature fiscale. Ils paient des impôts plus élevés selon un système de discrimination hérité des empires perse et byzantin. Il semblerait que le poids ait été élevé pour les couches les plus pauvres de la société. Ils devaient en outre

\footnotetext{
1 Pavon Benito, Julia Poblamento, Altamedieval navarro, base socioeconomica del espacio monarquico, Pamplona Eunsa, 2001, p. 14 - 16
}

2, Burgo, Jaime del, Historia de Navarra, Madrid, Tebas, 1978. Jaray, p. 448 
s'acquitter de diverses taxes (octroi, douane...) à des tarifs plus élevés que les Musulmans ${ }^{3}$. Mais ils jouissaient habituellement d'une certaine autonomie: ils possédaient leurs chefs et leurs juges et pouvaient mener leur vie familiale, personnelle et religieuse selon leurs propres lois. Car la loi coranique assure un statut particulier à tous les croyants de religions révélées (gens du livre). Les révoltes de Musa ibn Musa contre l'émir de Cordoue se répètent et sont associées à l'accord d'Arista de Pampelune et selon le chroniqueur Ibn Haiyan, les liens de famille très étroits les conduisaient à la même politique ${ }^{4}$. "Enneco Arista engendra Garcia Iñéquez et doña Assona qui fut l'épouse de Don Musa, maître de Borja et de Tenero..." 5

Par ailleurs, le système communautaire ${ }^{6}$ est poussé à l'extrême dans les cités de l'Islam. Il faut dire que les prescriptions du Coran consacrées aux relations entre Musulmans et non Musulmans sont nombreuses. L'Islam aboutit à une division en trois groupes: les Musulmans, les gens du livre et les idolâtres. Cette dernière catégorie est exclue de la tolérance pluraliste dont jouissent les gens du livre. Ces dernières bénéficient de la dhimma, contrat de protection octroyé par les Musulmans aux gens du livre, en contrepartie du paiement de la Jizya ou capitation. Aux yeux de Mohammad. Juifs, chrétiens et Musulmans descendent tous d'Abraham. Les gens du livre, après de vains essais de conciliation dogmatique, sont rejetés dans la catégorie des Infidèles sur le plan religieux, mais leur permanence est admise au sein de la communauté en hommage au Prophète et aux révélations antérieures. Ils devront cependant être humiliés et payer leur admission dans la communauté des croyants qui respectera leurs cultes, leurs croyances et leur autonomie juridictionnelle. Le traitement des Dhimmis dans la cité Musulmane s'est dégradé car la présence des communautés chrétiennes a souvent été ressentie comme un danger du fait des relations entretenues avec les puissances chrétiennes. La tolérance des Dhimmis imposée par le Prophète dans le Coran, a

\footnotetext{
3 Lewis, Bernard, Juifs en Terre d'Islam, Paris, Calmann-Lévy, 1984, p. 42-43.

${ }_{4}^{4}$ Arbeloa, Joaquin, Los origenes del Reino de Navarra, San Sebastian, ed. Auñamendi, 1969, vol. II, p. 404.

5 Traduction du Codice de Roda, Zaragoza, 1945, étudié par José Lacarra et cité par Narbaitz Pierre, Nabarra, ou quand les Basques avaient des rois, Pampelune-Bayonne, Zabal, 1978, p. 34 (637 p.)

${ }^{6}$ Corm, Georges C., Histoire du pluralisme religieux dans le bassin méditerranéen, Paris, Société Nouvelle, Librairie orientale, Paul Geuthner S.A. 1998, (1971) p. 129-244, 321 p.
} 
pour but à la longue d'amener ceux-ci à la vraie religion en leur permettant de constater tous les bienfaits du régime islamique. Certains califes exercent des pressions pour leur conversion, d'autres s'efforcent de mettre un frein pour ne pas faire baisser les entrées d'argent provenant du tribut.

\section{Musulmans et Navarrais}

Avant l'an mille, les rapports entre les royaumes se font sous la forme d'alliances matrimoniales, les souverains chrétiens du Nord de la péninsule et les chefs Musulmans étaient unis par des liens familiaux. Les mieux connus sont ceux qui liaient les souverains de Pampelune aux Banu Qasi installés à l'ouest autour d'Ejea. Les sources arabes permettent également de connaître les mariages qui eurent lieu entre souverains omeyyades et princesses navarraises. Los Banu Qasi de Tudela sont des Wisigoths convertis lors de la conquête.

En 839, Fortun Ibn Musa, fils du chef des Banu Qasi va défendre Medinaceli contre Alphonse. Les invasions Musulmanes se répètent presque tous les ans dans les zones frontières d'Alava et de Castille. Au IX siècle, ce nom apparait dans les chroniques de Lucas de Tuy ${ }^{7}$, de Jiménez de Rada et dans la Chronique d'Alphonse le Sage. Ce territoire fait partie des possessions qu'Alphonse le chaste conquit sur les Maures. Garcia II Iñiguez (860882), roi de Pampelune, est allié de Muza Ben Muza par qui il est tué, mais dès le début de son règne, il ferme les frontières d'Alava avec les châteaux de Zaldiaran et les Conchas de Arganzon. Mais les attaques sont incessantes.

Les premiers rois de Navarre utilisent leur force pour maintenir leur indépendance par rapport au royaume musulman voisin. Dans cette lutte, Garcia Inéguez fut tué par les soldats ennemis (852-882). En l'année 867, Mohamed de Cordoue, successeur d'Abderraman II, avec une armée puissante aux ordres d'Almondhir, franchit l'Ebre et parcourt la campagne de Navarre pendant trente-deux jours. Trois châteaux furent conquis: Firus, Falah'san et H'ahtir; dans ce dernier se trouvaient les enfants du roi, Fortun et Iñiga qui furent emmenés captifs à Cordoue. L'infante se maria avec le roi Abd Allah, fils de Mohamed, et fut la grand-mère d'Abderraman III. Fortun, quand son beau-frère Abd Allah monta sur le trône, retrouva sa liberté après vingt ans de captivité. Et Fortum Garces (882-905) passa vingt ans de sa vie à Cordoue avec sa sœur. Mais ce fut Sancho Garces (905-925) qui forgea le royaume de Navarre en s'opposant aux Musulmans. Il arriva à reconquérir les terres riches occupées par les Banu Qasi ${ }^{8}$, et ce fut alors que l'Ebre servit de

\footnotetext{
${ }^{7}$ Lucas Obispo de Tuy, Cronica de España, Madrid, ed. Romanceado, 1926, p. 444
} 
frontière après la conquête du château de Monjarden, de Los Arcos, Andosilla, Carcar et Milagro. Mais en 921, Abderraman III envahit la Rioja et s'opposa aux gens d'Alaba, du Guipuzcoa et de Navarre alliés. En 921, les Basques et les Lonais s'unissent contre les Musulmans à la bataille de Valdejunquera ${ }^{9}$.

En l'an 738, une garnison Maure occupe déjà Pampelune et pendant tout le viII siècle, le pouvoir alterne entre les autochtones et les envahisseurs. Les habitants se trouvant sur la frontière avec les terres d'Alava luttent contre les conquérants. En 778, Charlemagne traverse les Pyrénées pour attaquer Saragosse en passant par Pampelune ${ }^{10}$. Les Banu Qasi, descendants de Cassius, étaient les maîtres des rives de l'Ebre. Ils établirent des relations de parenté avec certaines familles Musulmanes de la frontière. L'émergence du royaume de Navarre surgit de son indépendance progressive vis-à-vis du royaume d'Aragon et de la puissance Musulmane ${ }^{11}$.

Iñigo Ximenez Aritza (824-852), premier roi des Basques suit une politique d'alliances avec les Maures et les Aragonais. Il donne en mariage ses filles à un Sarrazin afin d'obtenir des troupes pour combattre les Francs. En 841, Abderraman II de Cordoue envoie une puissante armée, mais Muza, petit-fils d'Aritza, s'allie aux Basques et soulève les Maures de Tudela. Abderraman fait le siège de la ville et son fils Mohamed va jusqu'à Pampelune.

Garcia I fut tué par une troupe Maure et il fut l'époux de doña Urraca, fille unique du comte d'Aragon Fortun Giménez, selon certaines sources. Les walis de Saragosse, Tudela et Valtierra mettent en péril les villages. En 981, le château Silvanio est détruit par Mohamed ben Lujo et l'année suivante, c'est le tour de la forteresse d'Aibar.

En 907, pendant que Sancho Garcès (905-926) se trouvait au nord des Pyrénées, les Maures assiègèrent Pampelune. Il revint pour défendre sa capitale et tua Lob ben Mohamed wali de Valtierra, arrière-petit-fils du Maure Muza.

En 910, ce roi navarrais fait la conquête de Mendavia, Lodosa,

\footnotetext{
8 descendants du comte Casius converti qui occupent les régions de Borja, Tarazona et Tudela, Arbeloa, 1969, I, 43.

${ }^{9}$ Tanco Lerga, Jesus, Navarra geografia e historia, Pamplona, ed. Gomez, 1972. p. 24-27

10 Tanco Lerga, Jésus, ibid. p. 19-20

11 Historia general del pais vasco, vol IV, Bilbao, ed la gran enciclopedid vasca p. 12-38, 81, dirigida par Julio Caro Baroja
} 
Carcar, San Adrian, Andosilla et Milagros, et repousse les Sarrasins jusqu'à l'Ebre. Il fait détruire les mosquées et construit des églises. Sancho el Craso neveu du roi Don Garcia de Pampelune se voit refuser le trône de Léon en 955. Il s'en va en territoire Musulman et revient avec une armée. En 966, Abderraman ben Jahya pénètre en Navarre et rase les zones frontières. Le prince Don Ramiro, deuxième fils du Roi Don Garcia est vaincu.

En 976, Aliatan, fils d'Abderraman meurt et Hixenâgé de 10 ans devient souverain, avec comme premier ministre le wisir Mohamed de 37 ans, secrétaire privé de Sobeya, épouse favorite du monarque décédé. On l'appelle Almanzor le victorieux, car en 26 ans, il réalise 52 expéditions contre les Chrétiens. A sa cour il reçoit le comte Vela de Castille persécuté par Fernan Gonzalez. La Navarre s'allie à la Castille pour vaincre les troupes maures. Ils prennent les places fortes de Gormaz et Atienza. En 985, Sancho Albarca revient pour vaincre les Maures qui font le siège de Pampelune.

Pendant le règne de Sancho Garcès III (999-1035), les luttes entre deux partis augmentent parmi les Maures. D'une part les Gacis avec à leur tête Soleiman venu d'Afrique pour les guerres, et ceux de Mohamed natifs d'Al Andalous. Ce dernier, ayant la faveur du roi Hixen, garde le pouvoir. Ils envahissent la Navarre par le Moncayo. Le chef, après son échec est décapité et le monarque doit prendre la fuite.

En 1045, Don Garcia Sanchez V (1035-1054), fait la guerre aux Maures et libère Calahora de 124 ans de domination maure. Il prit Tudela et fit payer l'impôt aux petits rois de Huesca et de Saragosse. Depuis 1046, le roi de Saragosse devait payer 12000 mancusos par an au roi de Navarre.

Par le jeu des mariages, des alliances multiples se créent des liens entre la Castille Leon, la Catalogne, l'Aragon et la Navarre qui se font et défont au gré des circonstances, car les chrétiens, après avoir été soumis par les Musulmans, n'ont d'autre but que de les soumettre à leur tour à des impôts qui leur permettront d'assurer leur pouvoir et donnent de la force au royaume de Navarre qui alors pourra se tourner vers les Francs.

La mise en place du pouvoir musulman est mal connue, et les auteurs arabes se réfèrent le plus souvent à l'ensemble de la vallée de l'Ebre, mais au VIII ${ }^{\mathrm{e}}$ siècle, le chroniqueur Al Udri utilise peut-être les annales de la marche. Les Banu Salama se rebellent contre Cordoue et prennent leur indépendance à Huesca. Mais un affrontement entre grandes familles musulmanes (IXème - Xème) ${ }^{12}$ persiste, et des rapports existent avec les souverains chrétiens voisins, de la franche collaboration à la lutte ouverte. La présence du pouvoir cordouan se manifestait par l'envoi de troupes. La Taïfa à laquelle la région 
de Huesca se trouvait intégrée était l'une des plus prospère, et les Banu Hud étendaient leur pouvoir jusqu'à la vallée de l'Ebre, aux confins de la Navarre et de l'Aragon. En 1046, Sulayman Ibn Hud, plaça ses cinq fils à la tête des villes principales. Tudela revint à Mundir et Ahmed demeura à Saragosse pour succéder à son père ${ }^{13}$.

Les sources arabes mentionnent les mariages qui eurent lieu entre les souverains omeyyades et les princesses navarraises. En 872, Mutarrif ibn Musa (Aragon) épouse Velazquita fille du souverain de Pampelune. Plus tard, Sancha, fille du comte Aznar Galindez II, épouse Muhammad al Tourl et lui donne quatre fils. La mère du gouverneur de Huesca était chrétienne, elle s'en retourna à Pampelune et épousa le roi Garcia Sanchez 1er 14. Les relations diplomatiques et alliances diverses étaient aussi fréquentes que l'échange d'objets, sans que l'on puisse affirmer que le commerce soit développé. Cependant, J.M. Lacarra a démontré que la marche supérieure fut une voie de passage entre l'Occident chrétien et le monde islamique. Les Chrétiens fournissaient bois, fourrures, peaux et minerais en échange de produits de luxe ${ }^{15}$. Cette évolution de l'occupation du terrain va de pair avec des alliances matrimoniales dont le mariage entre La Vascona, sœur de Garcia Sanchez II el Tembloroso (994-1004) et petite fille de Sancho Garcès II Abarca (970-994) avec Almanzor semble une phase importante ${ }^{16}$ avant les alliances avec les dynasties françaises. Cependant, la coupure se préparait déjà avec $\mathrm{Al}$. Andalous sous Sancho Garcès (905-925) qui rêvait d'expansion territoriale et religieuse ${ }^{17}$.

Les Musulmans dominent les bords de l'Ebre du VIIIème au IXe siècle, puis sous le pouvoir chrétien ils se regroupent dans les quartiers spécifiques désignés sous le nom d'aljamas ou morerias, enfin, dans la dernière

12 Les Banu Qasi, descendants du comte Casius (installés dans la région d'Ejea et convertis). Les Banu Amrus, descendants d'Amrus et Sabrit, convertis. Les Banu Tugibi, d'origine arabe (Saragosse, Daroca).

13 Laliena, Carlos, Sénac, Philippe, Musulmans et chrétiens dans le haut Moyen-Age aux origines de la reconquête aragonaise, Montrouge, Minerve, 1991, p. 13-32

${ }^{14}$ Levi Provençal, "Dù nouveau sur le royaume de Pampelune au IXè siècle", Bulletin hispanique, vol LV, 1953, p. 5-22

15 Laliena Carlos, Sénac Philippe, Musulmans et chrétiens dans le haut Moyen-Age aux origines de la reconquête aragonaise, ed. Minerve, p. $140-141$

${ }^{16}$ Landa El Busto, Luis, Historia de la Navarra, Pamplona, Fondo de Publicaciones del Gobiemo de Navarra, 1999, 332 p. (voir carte)

17 Généalogie des rois de Navarre IX-XIПè 
phase de l'occupation du territoire en Navarre, ils se regroupent dans des villages ou des bourgs comme Corella, Cascante, Tudela ou se trouve plus de la moitié de la population musulmane ${ }^{18}$.

L'étroite amitié entre la famille Arista et le clan des Banu Qasi protège les premiers des attaques musulmanes mais les soumet aux aléas de la politique de leurs alliés. Jusqu'en 841, les Banu Qasi ont de bonnes relations avec l'émir et Pampelune jouit de la paix, les expéditions musulmanes se dirigent vers l'Alaba. Mais lorsque deux nouveaux walis sont nommés à Saragosse et à Tudela, la situation change et les attaques se multiplient contre les possessions des Arista et des Banu Qasi ${ }^{19}$. Ces derniers s'unissent dans la guerre (déjà liés par des liens de famille) contre Harit ibn Bazi, wali de Saragosse, capturé lors de son passage de l'Ebre. Ces circonstances provoquent la campagne d'Abd al. Rahman II en mai 842, contre Pampelune qui déboucha sur un pacte: Iñigo Arista devait payer 700 dinars annuels d'impôt et rendre les prisonniers. Mais la rupture du pacte par le camp Arista provoque l'année suivante une nouvelle campagne, à l'issue de laquelle les chrétiens furent vaincus et, selon le chroniqueur Ibn Hayyan, nombreuses furent les personnalités de Pampelune qui passèrent au camp de l'émir et demandèrent sa protection 20 .

La relation de protégé (dhimmi) passe donc à travers les siècles de la position dominante Musulmans/Chrétiens à l'inverse Chrétiens/Musulmans dans un contexte totalement différent, mais le lien économique appuie toujours la situation religieuse.

\footnotetext{
18 Cortes, Monteagudo, Cintruénigo, ibid.p.73-75.

19 Lacarra, José Maria, Historia del Reino de Navarra en la Edad Media, Pamplona, Caja de Ahorros de Navarra, 1975, p. 277, p. 24, généalogie, p. 25. Famille Banu Qasi (Tudela), Iñigo Arista (Pamplona)

${ }^{20}$ Lacarra, ibid.p.26, généalogie p. 27, famille Jimena, Iñiga et Banu Qasi.
} 


\section{La Société}

En terre musulmane, la plus grande partie de la population était constituée par des descendants d'éléments indigènes convertis ou non à l'Islam. Et c'est le critère religieux qui permet d'appréhender cette société. Les descendants des premiers Arabes installés et des Berbères, étaient de plus en plus rares. L'essentiel de la population musulmane était constitué par des descendants de convertis à l'Islam, les muwalads poussés par des motifs d'ordre matériel.

L'Aragon et le royaume de Pampelune entretiennent des rapports de force et ce n'est pas par hasard si Sancho Garcès $1^{\text {er }}$ et son fils Garcia Sanchez $1^{\text {er }}$ choisirent comme lieu d'inhumation le château de San Esteban, localisé à proximité de l'Ebre. La présence de la dépouille du souverain dans cette position avancée, face aux fortifications musulmanes de Calahorra et de Valtierra, visait à protéger le royaume.

Les revenus tirés de la frappe monétaire n'étaient rien comparés aux tributs appelés parias. Ces versements imposés aux Musulmans, à la suite des victoires remportées par les Chrétiens, débutèrent peu de temps après la chute du califat de Cordoue. Mais ils ne devinrent importants et réguliers que dans la seconde moitié du XIè siècle, lorsque les Navarrais, Catalans et Castillans s'affrontèrent pour percevoir les parias de Saragosse et celles des autres principautés de la vallée de l'Ebre. En 1076, Sancho Ramirez acquit avec l'annexion de la Navarre les parias de Saragosse, que l'on peut estimer à 2000 dinars, En Navarre, à la mort de Sancho IV en 1076, l'absence d'un héritier direct entraîna la fragmentation du royaume.

En terre chrétienne, la aljama et la aljamia ${ }^{21}$ des Musulmans sont deux phénomènes de résistance à l'acculturation et l'éxil. La aljama est une communauté unie fiscalement devant l'autorité chrétienne qui doit se gérer selon ses lois religieuses et conserver ses croyances et son mode de vie. La aljamia est la langue romane que parlent les Musulmans. C'est dans la vallée de l'Ebre que vont rester des groupes importants de population sous la protection des seigneurs ou du roi ${ }^{22}$.

21 Langue pratiquée par les Musulmans en terre chrétienne, el aljamiado étant à transcription, mélange d'aragonais et d'arabe, écrit en caractères arabes.

22 Epalza, Mikel de "Caracterizacion del exilio Musulman: la voz de mudéjares y moriscos" in Destierros Aragoneses I Judios y Moriscos, Zaragoza, institucion Fernando el Catolico, 1988. p. $217-227$ 
La aljama est un quartier séparé extramuros, clôturé à Tudela, Corella, Cortes (morerias). Selon les accords avec Alphonse I, les Maures conservent leurs tribunaux et leurs juges et appliquent la loi islamique même dans les cas où des chrétiens sont compromis. Ils sont appelés alcadis et algalibos (lieutenant), alforques (chargés des impôts) et zalmedenia (responsable du commerce). Les jugements se rendent selon la sunna ou tradition islamique.

L'alcadi gère des relations avec la société chrétienne. Il écrit et parle l'arabe. En général il est nommé parmi les artisans du roi et cette charge constitue une récompense, mais elle n'intéresse pas les grandes familles. Certaines se spécialisent dans la charge de scribe et de notaire ${ }^{23}$ (en langue arabe) et paient pour cela un droit au roi. Souvent la charge de scribe et d'ulema (son remplaçant étant le çabiçala) sont rassemblées en une seule et même personne. El alamin à la charge de répartir les eaux d'irrigation avec la collaboration des zabacequias, et il prolonge les habitudes des siècles antérieurs ${ }^{24}$ et celles du monde Musulman. A ces responsabilités viennent s'ajouter celles de crieur public ${ }^{25}$ et de trésorier ${ }^{26}$. L'autorité du conseil de l'aljama s'affaiblit au cours des siècles vis-à-vis des conseils chrétiens et de l'Eglise.

Le bailly supervise les tribunaux Musulmans et se charge des arrestations nécessaires. Les Musulmans font appel au roi pour échanger des peines corporelles par des peines financières, lorsque la loi chrétienne leur est plus favorable.

A l'origine, la légitimité du pouvoir royal se confondait avec la lutte contre les Musulmans. La lutte contre l'Islam devient un projet dynastique jusqu'à l'expulsion. La domination religieuse et financière passe du pouvoir Musulman au pouvoir chrétien, jusqu'à la phase ultime de désertification théorique (l'assimilation des membres des communautés Musulmanes tombe dans l'oubli, mais ressort dans bien des régions lors de procès d'inquisition) et de la transformation des mosquées en églises.

\footnotetext{
${ }^{23}$ Actes d'achats, de ventes, contrats de mariage

24 Garcia Arenal Mercedes, Leroy Béatrice, Moros y judios en Navarre en la Baja Edad Media. Madrid, Hisperia, 1984, 286 p. p. 35-65

25 pregonero

26 clavero
} 
Des modifications importantes se sont produites à la fin du Moyen Age dans les communautés Musulmanes ou aljamas, situées à une zone frontière qui a subi de nombreuses fluctuations au cours des siècles, passant du pouvoir Musulman au pouvoir chrétien de la Navarre à l'Aragon.

- dans le domaine politique et administratif, l'organisation des aljamas se précise par rapport au pouvoir en place.

- dans le domaine économique, un système fiscal est mis en place au bénéfice du vainqueur.

- dans le domaine social, la participation à la vie publique et répartition de l'impôt évolue.

- dans le domaine des compétences, le haut niveau des artisans est reconnu par les chrétiens et même par le roi. Les confréries de travailleurs travaillent à des buts socio-religieux. 


\section{Chronologie}

\begin{tabular}{|c|c|c|}
\hline Pampelune & 738 & garnison maure \\
\hline Charlemagne & 778 & Saragosse - Roncevaux \\
\hline Iñigo Arista & +852 & \\
\hline Garcia Iñiguez & $852-882$ & \\
\hline Fortun Garces & $882-905$ & \\
\hline Sancho Garces & $905-925$ & $\begin{array}{l}\text { frontière: l'Ebre/union avec } \\
\text { l'Aragon }\end{array}$ \\
\hline Valdejunquera & 921 & défaite chrétienne \\
\hline Garcia Sanchez II & $926-970$ & \\
\hline Bataille de Simancas & 939 & défaite musulmane \\
\hline Sancho II Abarca & $970-994$ & $\begin{array}{l}\text { Almanzor/siège de } \\
\text { Pampelune }\end{array}$ \\
\hline Garcia Sanchez III el trémulo & $994-1004$ & Almanzor \\
\hline Sancho III el mayor & $1005-1035$ & $\begin{array}{l}\text { Taifas, extension vers } \\
\text { l'Aragon } \\
\text { rio Cinca, Sobrarbe, conda- } \\
\text { do de } \\
\text { Ribagorza } \\
\text { Alava, Guipuzcoa et } \\
\text { Viscaya }\end{array}$ \\
\hline Garcia III El de Najera & $1035-1054$ & $\begin{array}{l}\text { conquête de Calahorra sur } \\
\text { les Musulmans }\end{array}$ \\
\hline Sancho IV El de Peñalen & $1054-1076$ & $\begin{array}{l}\text { division des territoires chré- } \\
\text { tiens }\end{array}$ \\
\hline Sancho Ramirez & $1076-1094$ & $\begin{array}{l}\text { luttes avec le roi Maure de } \\
\text { Zaragosse } \\
\text { Al-Muktadir } \\
\text { conquête de Tolède en } 1085 \\
\text { par les Castillans } \\
\text { conquête de Cadreita par les } \\
\text { Chrétiens }\end{array}$ \\
\hline
\end{tabular}


Pedro I

1094-1104

(1100)

Alfonso I le batailleur

Garcia IV El restaurador

Sancho VI el sabio

Sancho VII el fuerte

Felipe III
1104-1134

1134-1150

1150-1194

1194-1234 conquête de Huesca sur les

Musulmans (1096)

conquête de Barbastro

conquête de Valtierra (1110)

+ d'Almustain, roi de

Zaragosse

conquête d'Ejea

conquête de Zaragosse

conquête de Tudela (1119)

séparation de la Navarre et de l'Aragon à sa mort

répartitions de territoires

conflits dynastiques

les Castillans et les

Aragonais souhaitent

partager le royaume de

Navarre

invasion almohade (1195)

soumission des Almoravides

invasion par les Chrétiens

voisins du royaume

de Navarre

16 juillet 1212 - Navas de Tolosa

persécution des Juifs 27

1516 expulsion des

Musulmans, désertification

des villages

27 Tanco Lerga, p. 30-65 